\title{
Decoupled inputs sliding mode controllers for a fuel cell-supercapacitor module in hybrid generation applications
}

\author{
Jerónimo J. Moré ${ }^{1}$ (D) Paul F. Puleston ${ }^{1} \cdot$ Enric Fossas $^{2} \cdot$ Cristian Kunusch $^{3}$
}

Received: 11 December 2018 / Accepted: 13 May 2019 / Published online: 24 May 2019

(c) The Author(s) 2019

\begin{abstract}
The development of Multiple Input/Multiple Output (MIMO) sliding mode control setups for a fuel cell/supercapacitor module is presented in this paper. The main objective of the proposed controllers consists in simultaneously satisfying the demand and regulating the DC bus voltage, even in the presence of model uncertainties and strongly varying operating conditions. Two design approaches are utilized to synthetise different control setups, each one capable to robustly deal with such control challenges: on one hand, variable-gains first-order sliding mode and, on the other, supert-wisting second-order sliding mode control. The stability of the nonlinear controlled system is formally analysed. Extensive simulations are conducted, to comparatively assess the performance of the proposed MIMO sliding mode controllers. Both control setups exhibited highly satisfactory results, demonstrating robustness to external disturbances and parameter variations, proving to be more suitable than classic linear PID controllers.
\end{abstract}

Keywords Fuel cell $\cdot$ Supercapacitors $\cdot$ Hybrid system $\cdot$ Sliding mode control

\section{Introduction}

During the last years, hybrid electric power generation systems based on renewable sources have been strongly studied and developed all over the world. In this sense, Fuel Cell (FC)-based systems result a promising choice due to its high efficiency, zero pollution, and sustainability [1-3]. In particular, the Proton-Exchange Membrane (PEM) FC-based systems are being extensively studied for transport [4] and stationary applications [5].

One of the main problems associated with the PEM FC is that abrupt changes in its current must be avoided, to prevent irreversible damages to the membranes, improve the $\mathrm{H}_{2}$ consumption, and reduce voltage drop transients [6]. For this reasons, FC are often complemented with a module capable to rapidly respond to fast load variations. To this end,

Jerónimo J. Moré

jmore@ing.unlp.edu.ar

1 Instituto LEICI, Facultad de Ingeniería, Universidad Nacional de La Plata and CONICET, La Plata, Argentina

2 Institut IOC and Department of Automatic Control, Universitat Politècnica de Catalunya, Barcelona, Spain

3 Brose Fahrzeugteile GmbH, Ohmstraße 2a, 97076 Wũrzburg, Germany supercapacitors (SC) show to be an efficient solution [7]. These FC/SC modules are frequently combined with renewable energy sources and a hydrogen production device to conform an auto-sustainable zero-emission Hybrid System (HS). Hydrogen production may be achieved then by taking advantage of the excess power generated by the renewable modules [8].

In an external control level, to optimize the HS performance as a whole, several supervision and energy management strategies that ensure effective operation have been implemented (e.g., [9-11]). Then, in an inner level, to fully control the HS power converters, diverse Sliding Mode (SM) techniques have proven to be adequate. Through a proper design, these techniques are able to provide excellent dynamic behaviour, robustness, and finite time convergence [12-14]. For instance, in [15], SM techniques are used for the control of the converter of a single fuel cell stack. In [16], a control strategy based on a linear PI and SM combination is presented for a FC/SC hybrid module. In [17], the control of a stationary autonomous hybrid electric power system is assessed, considering a relative degree approach and inductive loads, while [18] deals with a similar case, but applied to hybrid electric vehicles.

In such context, the authors have developed in [19] a sliding mode control setup for a FC/SC-based hybrid module topology 
like the one tackled in the present paper. In that preliminary work, a double SISO design approach has been followed to synthetize two complementary fixed-gains First-Order Sliding Mode (FOSM) controllers. The successful results obtained showed the suitability of sliding mode to robustly deal with this topology, in the presence of exacting operating conditions, such as highly variable load demand and varying wind.

Those encouraging results have thrusted the endeavours, leading to the enhanced control proposal presented in this article. Several new contributions have been developed. Among them, a diffeomorphism is utilized to deal with the inherent coupling of the system, allowing a MIMO more-efficient design approach. In addition, chattering reduction is attained. This undesirable phenomenon, particularly present in classic first-order sliding mode controllers, is successfully mitigated by developing a Second-Order Sliding Mode (SOSM) control setup. Another important issue, the stability of the nonlinear controlled system, is established by providing a formal analysis of its zero dynamics, which was omitted in [19].

Summarizing, this paper addresses the development of two different MIMO control setups for the FC/SC module, which is assumed to be part of an already existent HS that also comprises renewable power sources and an electrolyzer. Both proposed MIMO controllers, one based on variable-gain FOSM and the other on SOSM, prove to be robust. Simulation results considering parameter variations and variable load power demand are thoroughly assessed and discussed, including comparisons between both MIMO setups and with a classic PID control structure.

\section{System description}

\section{Model of the $\mathrm{FC} / \mathrm{SC}$ module}

A schematic diagram of the HS is shown in Fig. 1, where the FC/SC module is highlighted by dashed lines. The fuel cell stack and the supercapacitors bank are connected to a DC Bus via boost converters. In addition, the electrolyzer and a renewable energy source (in this case, a wind energy conversion system (WECS)) are also displayed, to provide a complete representation of a HS.

It is assumed that the power references for the controllers of each module are computed, at a higher level, by an external supervisory control that coordinates the power flows interaction over the entire system.

Figure 2 presents the circuit diagram of the FC/SC module. It can be modelled by the following control affine equations:

$\dot{x}=f(x, t)+g(x) \cdot u$,

where the state vector results

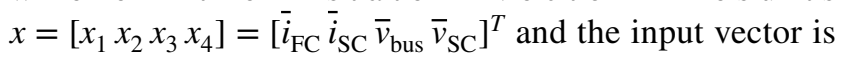
given by $u=\left[u_{1}=1-d_{1} u_{2}=1-d_{2}\right]^{T}$, with $d_{i}$ the duty

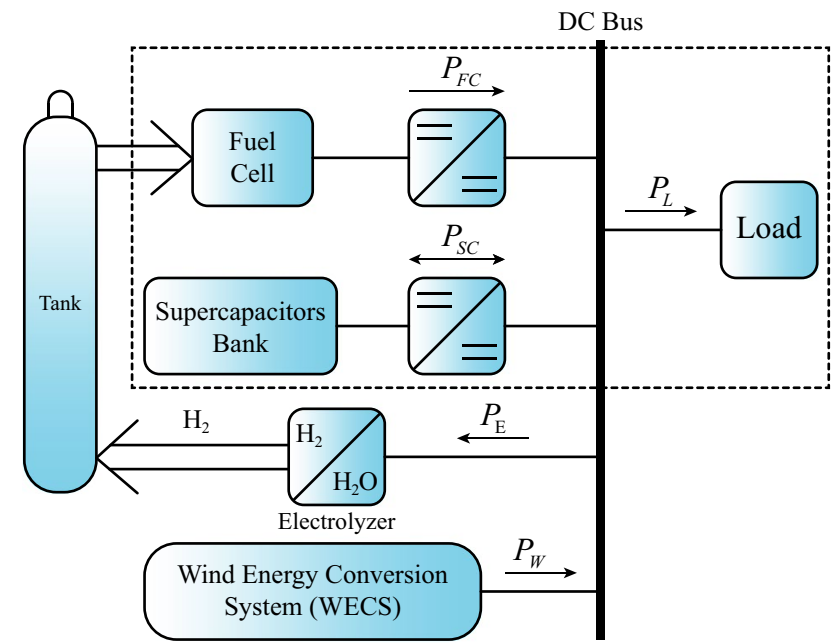

Fig. 1 Schematic diagram of FC/SC module embedded in the HS

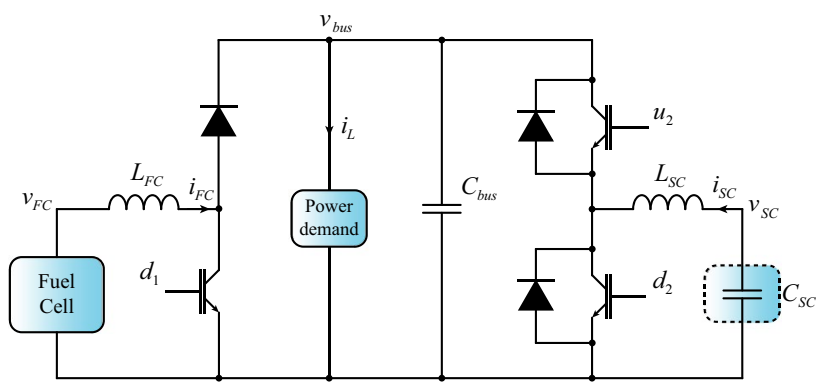

Fig. 2 FC/SC module equivalent circuit diagram

cycle of the $i$ th converter switch, as indicated in Fig. 2. Matrix functions $f(x, t)$ and $g(x)$ are

$f(x, t)=\left[\begin{array}{c}\frac{v_{\mathrm{FC}}\left(x_{1}\right)}{L_{\mathrm{FC}}} \\ \frac{x_{4}}{L_{\mathrm{SC}}} \\ -\frac{L_{L}(t)}{C_{\text {bus }}} \\ -\frac{x_{2}}{C_{\mathrm{SC}}}\end{array}\right] ; g(x)=\left[\begin{array}{cc}-\frac{x_{3}}{L_{\mathrm{FC}}} & 0 \\ 0 & -\frac{x_{3}}{L_{\mathrm{SC}}} \\ \frac{x_{1}}{C_{\text {bus }}} & \frac{x_{2}}{C_{\text {bus }}} \\ 0 & 0\end{array}\right]$,

where $i_{L}(t)$ stands for the time varying load current and

$\begin{aligned} v_{\mathrm{FC}}\left(x_{1}\right)= & N_{\mathrm{FC}} \cdot\left(E_{\mathrm{oc}}-R_{\mathrm{FC}} \cdot x_{1}\right. \\ & -A \cdot \log \left(x_{1}\right)-m \cdot \exp \left(n \cdot x_{1}\right),\end{aligned}$

represents the FC output voltage as a function of the stack current. In addition, $\bar{i}_{\mathrm{FC}}, \bar{i}_{\mathrm{SC}}$ are the FC and SC currents; $\bar{v}_{\text {bus }}, \bar{v}_{\mathrm{SC}}$ are the DC bus and SC voltages; $C_{\mathrm{bus}}, C_{\mathrm{SC}}$ are the DC bus and SC capacitances; $L_{\mathrm{FC}}, L_{\mathrm{SC}}$ are the FC and SC power converter inductances; $N_{\mathrm{FC}}$ is the cell number in the FC stack; $E_{\mathrm{oc}}$ is the FC open circuit voltage; $R_{\mathrm{FC}}$ is the FC 
linear resistance; $A$ is the Tafel equation constant; and $m, n$ are the constants of the mass transfer overvoltage.

\section{Supervisory control strategy}

This section briefly sums up a supervisory control strategy that is assumed to compute the coordinated power references for each module of the HS under consideration. The supervisory control is considered to be existent, and hence, it is not of interest of this paper to go in-depth on this strategy. Details can be found in [19]. The main objective of the HS is to adequately satisfy the external load demand at regulated voltage. In addition, ancillary objectives are to maintain the SC bank adequately charged and to generate hydrogen to refill the tank, provided that spare power is available from the alternative energy source (e.g., the WECS). The supervisory control strategy is schematically summarized in the flowchart of Fig. 3.

From this figure, $P_{\text {tot }, r}$ is the total power demand in the DC bus; $P_{\mathrm{L}}$ is the load power demand; $P_{\mathrm{E}}$ and $P_{\mathrm{E}_{\mathrm{ref}}}$ are the electrolyzer actual power and its reference; $P_{\mathrm{W}}, P_{\mathrm{W}_{\text {ref }}}$ and $P_{\mathrm{W}, \text { max }}$ are the WECS actual power delivered to the DC bus, the WECS power reference, and the maximum available wind power, respectively; $P_{\mathrm{FC}}$ and $P_{\mathrm{FC}_{\text {ref }}}$ are the FC actual power and its reference; and $P_{\mathrm{SC}_{\text {ref }}}$ is the SC power reference, obtained from the power balance in the DC bus.

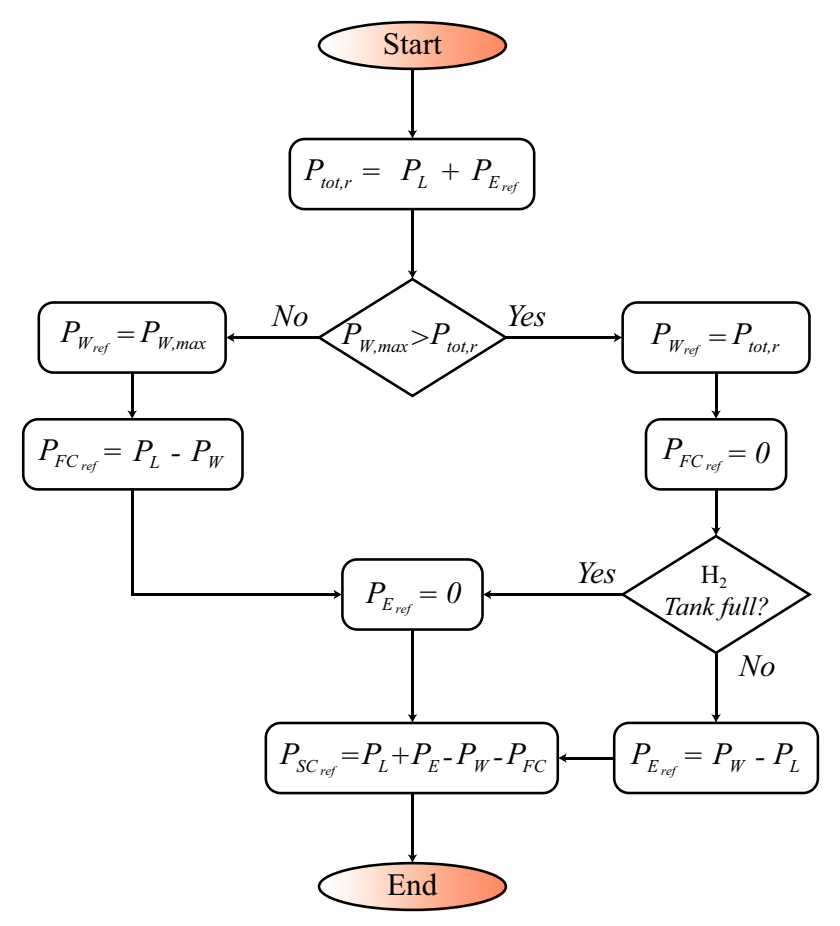

Fig. 3 Supervisory control strategy flowchart

\section{FC/SC module SM controllers design}

The FC/SC module control main objectives are to guarantee a constant DC bus voltage and to satisfy the module's power demand, even under heavy load variations.

Besides, some practical restrictions on the FC current must be taken into account. In addition to a maximum admissible value for that current, it is also convenient to impose a bound to the FC current slew rate. Three main reasons can be cited to support the latter. Firstly, the inherent stack dynamics limits the velocity at which power can be delivered to the load, i.e., FC's power cannot be set arbitrarily fast. Secondly, fast current changes may produce thermal stress at the catalyst surface, reducing the membrane lifetime. Finally, an adequate slew rate limitation may contribute to a fuel cell hydrogen consumption reduction [20].

To overcome such constraints, the FC is complemented by the SC to accurately maintain the power balance. This requires the $\mathrm{SC}$ converter to act rapidly to reject abrupt load variations and provide the so called peak shaving capability. Once the fast transient is extinguished, the control must enable the SC bank to recharge, slowly, to avoid FC overload.

Accordingly with the SM control theory, this set of control objectives must be formalized into the construction of suitable sliding variables $S=\left[s_{1} s_{2}\right]^{T}$, comprising current references based on the power references computed online by the supervisory control. The objectives are successfully attained when the SM controllers establish sliding mode regimes on the surfaces defined by $S=0$.

\section{Objective 1 into fuel cell sliding variable}

The FC converter sliding variable is designed to follow smooth load power variations, considering the FC current limitations mentioned in the previous section. In steady state, the fuel cell stack must supply the power required by the supervisory algorithm. In addition, the FC should recharge the SC bank to a desired value if needed. The above-mentioned requirements are fulfilled by the following sliding variable structure:

$s_{1}(x)=x_{1}-I_{\text {TotalFC,ref }}$

$=x_{1}-\left[I_{\mathrm{FC}, \text { ref }}-a_{1} \cdot\left(x_{4}-V_{\mathrm{SC}, \text { ref }}\right)\right]$,

with $I_{\text {TotalFC,ref }}$ a lumped FC current reference that is computed in accordance with the aforementioned limitations for the slew rate and maximum FC current; $a_{1}>0$ a design gain of the sliding surface. This gain must be selected considering the desired SC bank charging dynamics. Small values for $a_{1}$ result in a slow recharging dynamics, while higher values imply faster SC-charging times. 
$V_{\mathrm{SC}, \text { ref }}$ is the $\mathrm{SC}$ voltage reference and $I_{\mathrm{FC} \text {,ref }}$, the first term of $I_{\text {Total, FC,ref }}$, corresponds to the current that the FC has to deliver to supply the required power to the load. This current reference is computed as follows:

$I_{\mathrm{FC}, \mathrm{ref}}=\frac{P_{\mathrm{FC}_{\mathrm{ref}}}}{v_{\mathrm{FC}}\left(x_{1}\right)}$,

where $P_{\mathrm{FC}_{\text {ref }}}$ is the aforementioned FC power reference, computed by the supervisory control.

\section{Objective 2 into supercapacitors sliding variable}

The SC must deal with the high-frequency load changes and maintain the power balance. Thus, taking advantage of the fast dynamic response of the SC bank, its sliding variable is designed to regulate the DC bus voltage. In this case, a boost output voltage regulation surface is considered, combining both DC bus voltage and SC current:

$s_{2}(x)=x_{2}-I_{\mathrm{SC}, \text { ref }}+a_{2} \cdot\left(x_{3}-V_{\text {bus,ref }}\right)$,

where $V_{\text {bus,ref }}$ is the bus voltage reference; $a_{2}$ is a positive surface gain selected to obtain a desired closed-loop $x_{3}$ dynamics. $I_{\mathrm{SC} \text {,ref }}$ is obtained from the $\mathrm{SC}$ power reference, that is

$I_{\mathrm{SC}, \text { ref }}=\frac{P_{\mathrm{SC}_{\mathrm{ref}}}}{x_{3}}$

being $P_{\mathrm{SC}_{\mathrm{ref}}}$ the SC power reference obtained by the supervisory control.

\section{Control action design}

The proposed control actions are composed by two terms. The first one is a biasing nominal control action, responsible of steering the states to the vicinity of the switching surface. The second one, is a sliding mode control action, that will force the system to sliding regime operation and will robustly reject parameter variations, uncertainties, and external disturbances:

$u_{i}=u_{i N}+u_{i \mathrm{SM}}$.

It is important to remark that the former allows to reduce the sliding mode gains, which in turn helps to lessen the overall control effort and the chattering effect.

\section{Nominal control term design}

The nominal control terms are initially based on the equivalent control action [21]. Giving $S=\left[s_{1} s_{2}\right]^{T}$, the equivalent controls can be obtained by taking $\dot{S}=0$ and $S=0$ and solving for $u_{1}$ and $u_{2}$ :

$\dot{S}=\left[\begin{array}{l}\dot{x}_{1}+a_{1} \cdot \dot{x}_{4}-\dot{I}_{\mathrm{FC}, \text { ref }} \\ \dot{x}_{2}+a_{2} \cdot \dot{x}_{3}-\dot{I}_{\mathrm{SC}, \mathrm{ref}}\end{array}\right]=\left[\begin{array}{l}0 \\ 0\end{array}\right]$
$=\left[\begin{array}{c}\frac{v_{\mathrm{FC}}\left(x_{1}\right)}{L_{\mathrm{FC}}}-\frac{x_{3} \cdot u_{1}}{L_{\mathrm{FC}}}-\frac{a_{1} \cdot x_{2}}{C_{\mathrm{SC}}}-\dot{I}_{\mathrm{FC}, \text { ref }} \\ \frac{x_{4}}{L_{\mathrm{SC}}}-\frac{x_{3} \cdot u_{2}}{L_{\mathrm{SC}}}-\frac{a_{2} \cdot \cdot_{\mathrm{L}}}{C_{\text {bus }}}+\frac{a_{2} \cdot x_{1} \cdot u_{1}}{C_{\text {bus }}}+ \\ +\frac{a_{2} \cdot x_{2} \cdot u_{2}}{C_{\text {bus }}}-\dot{I}_{\mathrm{SC}, \text { ref }}\end{array}\right]$

Then, when $S=0$, the equivalent controls actions can be derived from

$u_{1, \mathrm{eq}}=\frac{v_{\mathrm{FC}}\left(x_{1}\right)}{x_{3}}-\frac{a_{1} L_{\mathrm{FC}} \cdot x_{2}}{C_{\mathrm{SC}} \cdot x_{3}}-\frac{L_{\mathrm{FC}} \dot{I}_{\mathrm{FC}, \mathrm{ref}}}{x_{3}}$

$$
\begin{aligned}
u_{2, \mathrm{eq}}= & \frac{C_{\mathrm{bus}} \cdot x_{4}-a_{2} L_{\mathrm{SC}} \cdot\left(i_{\mathrm{L}}-x_{1} \cdot u_{1, \mathrm{eq}}\right)}{C_{\mathrm{bus}} \cdot x_{3}-a_{2} L_{\mathrm{SC}} \cdot x_{2}} \\
& -\frac{C_{\text {bus }} L_{\mathrm{SC}} \dot{I}_{\mathrm{SC}, \text { ref }}}{C_{\text {bus }} \cdot x_{3}-a_{2} L_{\mathrm{SC}} \cdot x_{2}} .
\end{aligned}
$$

These equivalent control laws can be directly used as the nominal control terms in (9). In practice, however, aiming to reduce the online computational burden of the controllers, suitable simpler expressions for $u_{i N}$ can be obtained from approximated versions of (12) and (13), as follows.

In steady-state conditions, when there are no load variations, the current references for both converters are kept constant, so $\dot{I}_{\mathrm{FC}, \text { ref }}=0$ and $\dot{I}_{\mathrm{SC} \text {,ref }}=0$. In addition, in the same condition, when all the states have reached its reference values (i.e., $x_{1}=I_{\mathrm{FC} \text {,ref }}, x_{2}=I_{\mathrm{SC} \text {,ref }}, x_{3}=V_{\mathrm{bus}, \text { ref }}$ and $\left.x_{4}=V_{\mathrm{SC}, \text { ref }}\right)$, it can be shown that $I_{\mathrm{SC}, \text { ref }} \rightarrow 0$ and $x_{1} \cdot u_{1, \text { eq }} \rightarrow i_{\mathrm{L}}$. Considering this, the nominal control actions for both converters result as

$u_{1 N}=\frac{v_{\mathrm{FC}}\left(x_{1}\right)}{x_{3}}$,

$u_{2 N}=\frac{x_{4}}{x_{3}}$,

where $v_{\mathrm{FC}}\left(x_{1}\right)$ is a measurable variable of the system, whose measurement can be used for online implementation.

It should be emphasized that the objective of the nominal control actions $u_{i N}(i=1,2)$ is not to force the trajectories to reach the sliding surfaces, but to bring them to the region near to $S=0$. The sliding mode control actions designed in the sequel will be in charge of ensuring reaching and permanence on $S=0$.

\section{Sliding mode control action decoupling}

To simplify the design the sliding mode control inputs and switching conditions, an input to surface decoupling 
methodology is proposed. This objective is achieved by a suitable diffeomorphism defined as follows.

Be $T(x)$ a diffeomorphism that defines a new control action $w_{\mathrm{SM}}$ :

$w_{\mathrm{SM}}=T(x) \cdot u_{\mathrm{SM}}$,

where $w_{\mathrm{SM}}=\left[w_{1 \mathrm{SM}} w_{2 \mathrm{SM}}\right]^{T}$ and $u_{\mathrm{SM}}=\left[u_{1 \mathrm{SM}} u_{2 \mathrm{SM}}\right]^{T}$. Computing the time derivative of $S$ yields

$$
\begin{aligned}
\dot{S}= & \frac{\partial S}{\partial x} \cdot f(x)+\frac{\partial S}{\partial x} \cdot g(x) \cdot u+\frac{\partial S}{\partial t} \\
= & \frac{\partial S}{\partial x} \cdot f(x)+\frac{\partial S}{\partial x} \cdot g(x) \cdot u_{N}+ \\
& +\frac{\partial S}{\partial x} \cdot g(x) \cdot T(x)^{-1} \cdot w_{S M}+\frac{\partial S}{\partial t}
\end{aligned}
$$

where $u_{N}=\left[u_{1 N} u_{2 N}\right]^{T}$. Then, selecting the diffeomorfism $T(x)=\frac{\partial S}{\partial x} \cdot g(x)[22]$, equation (17) results

$\dot{S}=\frac{\partial S}{\partial x} \cdot f(x)+\frac{\partial S}{\partial x} \cdot g(x) \cdot u_{N}+I_{2} \cdot w_{\mathrm{SM}}+\frac{\partial S}{\partial t}$,

with $I_{2}$ the $2 \times 2$ identity matrix.

In this way, each control input $w_{i S M}$ acts only over the correspondent variable $s_{i}$, and both control actions can be designed independently. It is worth noting that the matrix $T(x)$, as defined, results:

$T(x)=\left[\begin{array}{cc}-\frac{x_{3}}{L_{\mathrm{FC}}} & 0 \\ \frac{a_{2} \cdot x_{1}}{C_{\text {bus }}} & \frac{a_{2} \cdot x_{2}}{C_{\text {bus }}}-\frac{x_{3}}{L_{\mathrm{SC}}}\end{array}\right]$

The inverse of this matrix always exists if $x_{3} \neq 0$ and $\frac{a_{2} \cdot x_{2}}{C_{\text {bus }}}-\frac{x_{3}}{L_{\mathrm{SC}}} \neq 0$. The former condition is fulfilled given that always exists a positive voltage at the DC bus. The latter condition is accomplished in the operation range by selecting the SC converter surface gain $a_{2}$ accordingly.

\section{Variable-gains first-order sliding mode design}

To attain sliding mode operation $(S=0)$, the following variable-gain FOSM control law is proposed:

$w_{i \mathrm{SM}}=K_{\mathrm{var}}\left(x_{i}\right) \cdot \operatorname{sign}\left(s_{i}\right)=\left(W_{c i}+W_{a i} \cdot\left|x_{i}\right|\right) \cdot \operatorname{sign}\left(s_{i}\right)$,

where $W_{c i}$ and $W_{a i}$ (with $i=1,2$ ) are design parameters of the variable gain $K_{\text {var }}\left(x_{i}\right)$. Note that the converter unmodelled losses are directly affecting its current dynamics, so the inclusion of the linear term in the gain $K_{\text {var }}\left(x_{i}\right)$ can largely mitigate their impact.

The design of gains $W_{c i}$ and $W_{a i}$ can be done by bounding the terms in (18) and conducting an error propagation analysis. In this case, the independence between both control actions $\left(w_{1 \mathrm{SM}}\right.$ and $\left.w_{2 \mathrm{SM}}\right)$ allows to use this method without any major complications. It must be remarked that the initial set of gains obtained through this method are usually too conservative.
For suitable application, further offline in-silico iterative tuning is required to simultaneously ensure the existence of the sliding mode regime while minimizing the output chattering. In this particular case, for the nominal system in the "Appendix", the obtained values of $W_{c i}$ and $W_{a i}$ were 5000 and 1000, respectively.

\section{Second-order sliding mode design}

In this section, a control algorithm that enforces $S=0$ and $\dot{S}=0$ is designed. For the relative degree one system under consideration, a super-twisting sliding mode control technique is used:

$\begin{cases}w_{i S \mathrm{SM}} & =W_{P i} \cdot \sqrt{\left|s_{i}\right|} \cdot \operatorname{sign}\left(s_{i}\right)+z_{i} \\ \dot{z}_{i} & =W_{I i} \cdot \operatorname{sign}\left(s_{i}\right) \mathrm{d} t\end{cases}$

where $W_{P i}$ and $W_{I i}$ are gains that need to be designed computing bounds based on $\dot{S}$ and $\ddot{S}$, and $i=1,2$. Particularly, Eq. (18) can be rewritten as

$\dot{S}=\phi\left(x, u_{N}, t\right)+I_{2} \cdot w_{\mathrm{SM}}$.

Then, $\ddot{S}$ results

$\ddot{S}=\Phi\left(x, u_{N}, t\right)+I_{2} \cdot \dot{w}_{\mathrm{SM}}$,

where

$\Phi\left(x, u_{N}, t\right)=\dot{\phi}\left(x, u_{N}, t\right)=\left[\begin{array}{c}\Phi_{1}\left(x, u_{N}, t\right) \\ \Phi_{2}\left(x, u_{N}, t\right) .\end{array}\right]$

From (23), it can be shown that if

$W_{I i} \geq\left|\Phi_{i}\left(x, u_{N}, t\right)\right|$,

$W_{P i}=2 \sqrt{W_{I i}}$,

the local convergence to the surface and the sliding motion is guaranteed. Note that for actual application, the values of the gains are tuned by extensive simulation, aiming to reduce the values computed from (25)-(26) and to obtain minimum chattering. In this particular case, they were set as $W_{P 1}=10,000, W_{I 1}=100,000, W_{P 2}=4000$, and $W_{I 2}=7000$.

\section{Stability analysis: zero dynamics}

With the sliding mode regime secured by control (9), the stability of the closed loop must be established by analysing the zero dynamics of the system. That reduced internal dynamics can be obtained from (1) by taking $S=0$, which imposes two algebraic restrictions over the states, and by replacing $u$ with the equivalent control actions $u_{\text {eq }}(12)-(13)$.

Then, the dynamics order is reduced from four, of the original system, to two, for the zero dynamics. In the case under 
study, it can be expressed in terms of the DC bus and the SC voltages, i.e., $x_{3}$ and $x_{4}$, respectively:

$\dot{x}_{3}=-\frac{i_{L}(t)}{C_{\mathrm{bus}}}+\frac{x_{1}}{C_{\mathrm{bus}}} \cdot u_{1, \mathrm{eq}}+\frac{x_{2}}{C_{\mathrm{bus}}} \cdot u_{2, \mathrm{eq}}$,

$\dot{x}_{4}=-\frac{x_{2}}{C_{\mathrm{SC}}}$,

where the states $x_{1}$ and $x_{2}$, and the equivalent controls $u_{1, \mathrm{eq}}$ and $u_{2 \text {,eq }}$, can be written as functions of $x_{3}$ and $x_{4}$, provided that $S=0$.

For the analysis, it must be considered the dependence of the $\mathrm{FC}$ voltage $\left(v_{\mathrm{FC}}\right)$ with the $\mathrm{FC}$ current $\left(x_{1}\right)$. In this way, a linear approximation of Eq. (3) is used, to simplify the following procedure. This approximation is valid assuming that the system operates in continuous conduction mode and such condition will make the FC to operate in its linear zone. The FC voltage can then be represented as

$\nu_{\mathrm{FC}}\left(x_{1}\right) \approx E_{\mathrm{oc}_{\mathrm{L}}}-R_{\mathrm{FC}_{\mathrm{L}}} \cdot x_{1}$.

Substituting these expressions and the nominal values of the system parameters (see "Appendix"), and assuming operating conditions, where $\dot{I}_{\text {FCref }}$ and $\dot{I}_{\text {SCref }}$ are neglectable, the second-order zero dynamics on $x_{3}$ and $x_{4}$ is obtained (the complete expressions of the zero dynamics in terms of $x_{3}$ and $x_{4}$ are not given due to space limitations).

A numeric study of the vector field allows to establish that $\left(x_{3}=V_{\text {busref }} ; x_{4}=V_{\text {SCref }}\right)$ is an equilibrium point of the system. In this case, the references values were set as $V_{\text {busref }}=75 \mathrm{~V}$ and $V_{\text {SCref }}=40 \mathrm{~V}$. As a first stability criterion, the first Lyapunov method is used. This method assess the eigenvalues of the Jacobian of the vector field:

$J_{\mathrm{ZD}}=\left.\left(\begin{array}{ll}\frac{\partial \dot{x}_{3}}{\partial x_{3}} & \frac{\partial \dot{x}_{3}}{\partial x_{4}} \\ \frac{\partial \dot{x}_{4}}{\partial x_{3}} & \frac{\partial x_{4}}{\partial x_{4}}\end{array}\right)\right|_{\left(x_{3}, x_{4}\right)=(75,40)}$.

Computing this Jacobian for the load current operation range always results in real and negative eigenvalues, with mean values of $\lambda_{1}=-3921.57$ y $\lambda_{2}=-5.54 \times 10^{-3}$. This shows the local stability of the equilibrium point. However, it is important to prove stability in a region that contains the whole operation range, to ensure the stability of the HS. To this end, in addition to the previous condition, it must be found an invariant region that includes the stable equilibrium point and the complete operation range. A diagram of the vector field around the equilibrium point and some representative solutions of the zero dynamics are depicted in Fig. 4. In this figure is shown that an invariant region can be defined considering the outer trajectories (in dashed-dotted

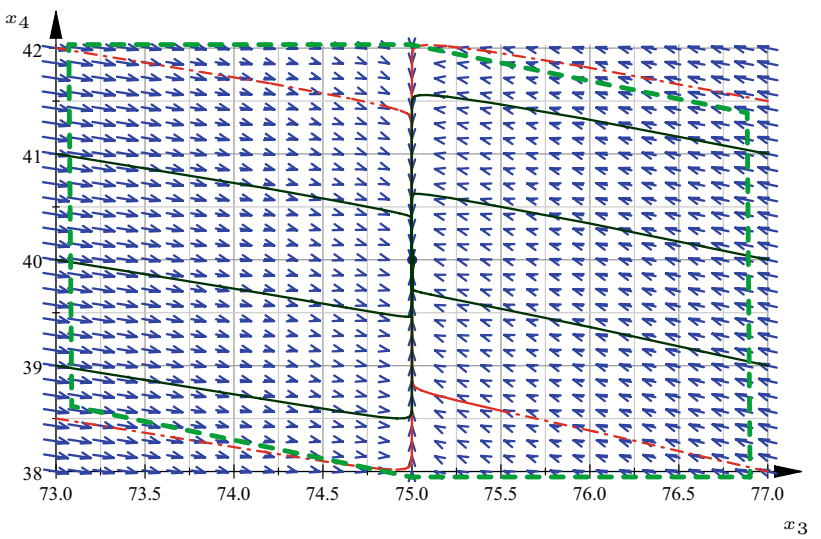

Fig. 4 Zero dynamics vector field and complete-range invariant region around the equilibrium point $(75,40)$

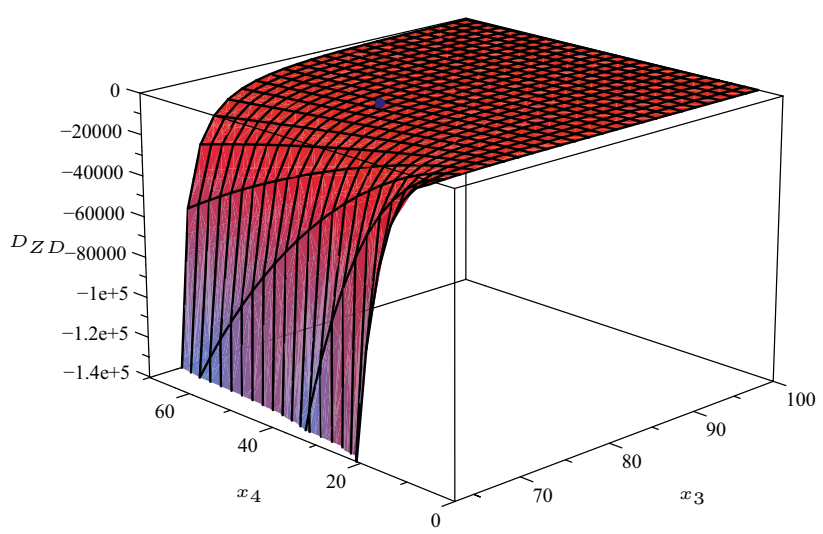

Fig. 5 Divergence of the zero dynamics vector field around the equilibrium point $(75,40)$

red). Specifically, it is established an invariant region within the dashed green area.

Proving the existence of the invariant region is still not enough to ensure that every trajectory of the system will converge to the equilibrium point. It must be also proven that there are no closed trajectories, or orbits, inside the invariant region. To do so, the Bendixson criterion is used, considering the divergence of the vector field. It is stated that if the divergence is not zero and does not change sign inside the region, the system has no orbits in that region. The divergence of the vector field is computed as

$D_{\mathrm{ZD}}\left(x_{3}, x_{4}\right)=\frac{\partial \dot{x}_{3}}{\partial x_{3}}+\frac{\partial \dot{x}_{4}}{\partial x_{4}}$.

A graphic of this expression around the equilibrium point is shown in Fig. 5. It should be noted that even though the divergence depends on the load current, the changes are 
practically negligible and do not affect the sign changes within the operation range.

It can be concluded then that, given the local stability of the equilibrium point and the existence of an invariant region, where the divergence of the vector field never changes sign, the zero dynamics results stable and converges to the references, within the operating region.

\section{Simulation results}

In this section, the performance of both proposed MIMO control setups, FOSM and SOSM, are assessed. For the analysis, extensive simulation were done, considering the FC/SC subsystem operating as part of the complete hybrid system, i.e., including the WECS, the electrolyzer, and a variable external load. As mentioned, the whole system is managed by a supervisory control that computes the power flow references for each module of the HS [19].

Realistic simulations were conducted using the complete full-order system model incorporating parameters variations up to $10 \%$ of their nominal values. In addition, highly variable power demand and available power of the WECS were considered, as depicted in Figs. 6 and 7 , respectively. The step-like load variations used for the tests are intended to force the system to operate under extreme conditions. Figure 7 also displays the wind power reference (assumed to be provided by the supervisory control) and the actual wind power delivered into the DC Bus by the WECS. The power consumed by the electrolyzer, resulting from those load and WECS power conditions, is shown in Fig. 8. Note that the load, wind, and electrolyzer powers, displayed in Figs. 6, 7 and 8, respectively, are externally given input variables from the FC/SC module viewpoint.

When the available wind power is not enough to satisfy the total power demand, the FC/SC subsystem must act to deliver the required power to the load and accurately regulate the DC bus voltage (Fig. 9). It can be appreciated that both control strategies (FOSM and SOSM) regulate this voltage efficiently, even when large power changes in load demand occur. A couple of zoomed views are included, displaying the start up of the system until the $75 \mathrm{~V}$ reference is reached and a perturbation rejection due to an abrupt load change. From this figure, it is important to notice that even though the first-order sliding mode accurately manage to regulate the DC bus voltage with low-voltage drop due to abrupt load variations, it has higher chattering. On the other hand, the second-order sliding mode has a minimum chattering and a faster recover from voltage drops.
Fig. 6 Hybrid system load power demand $P_{\mathrm{L}}$
Fig. 7 WECS maximum available power $\left(P_{\mathrm{W}, \max }\right)$, WECS power reference $\left(P_{\mathrm{Wref}}\right)$ and WECS actual delivered power $\left(P_{\mathrm{W}}\right)$
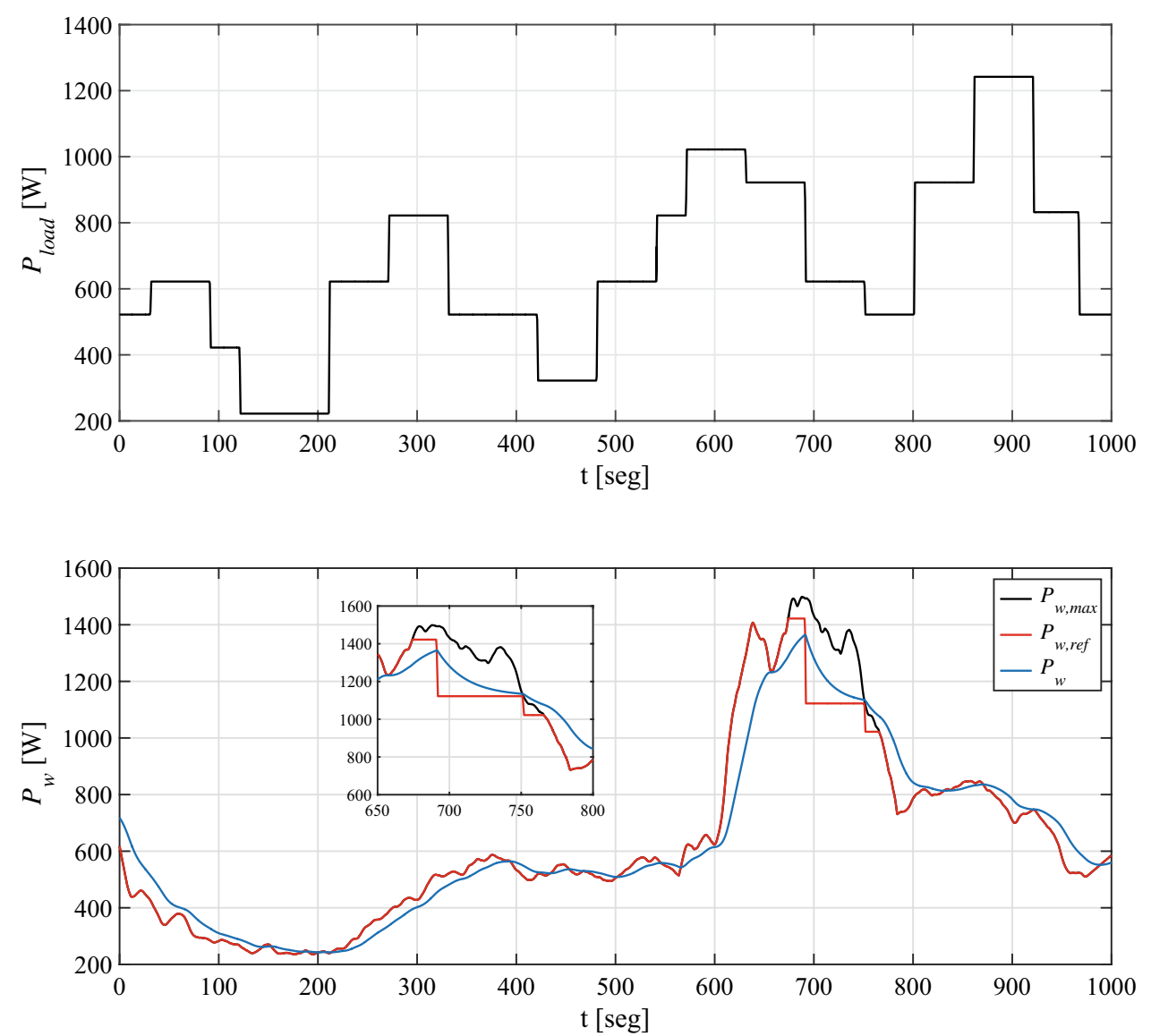
The FC voltage and current are shown in Figs. 10 and 11, respectively. From the latter, it can be appreciated that the current rate limitation imposed to the reference by the control strategy makes the system to slowly reach the different operation points and accurately supply the required power, in accordance with the load power demand variations. In addition, it can be again observed that the FOSM exhibits a higher chattering effect than the SOSM. It is important to remark that the FC current is never set to 0 , but to a minimum, to avoid stack star/stop process.

The voltage and current of the SC are presented in Figs. 12 and 13, respectively. In this case, the SC are smoothly recharged to its reference voltage (i.e., $40 \mathrm{~V}$ ), while the current quickly responds to abrupt load power demands, showing the excellent behaviour of the developed controllers. It can be appreciated that, mainly due to the chattering, the FOSM controller produces more power
Fig. 8 Hybrid system electrolyzer power consumption $P_{\mathrm{E}}$

Fig. 9 DC bus voltage $x_{3}=\bar{v}_{\text {bus }}$ regulation

Fig. 10 Fuel cell voltage $v_{\mathrm{FC}}$
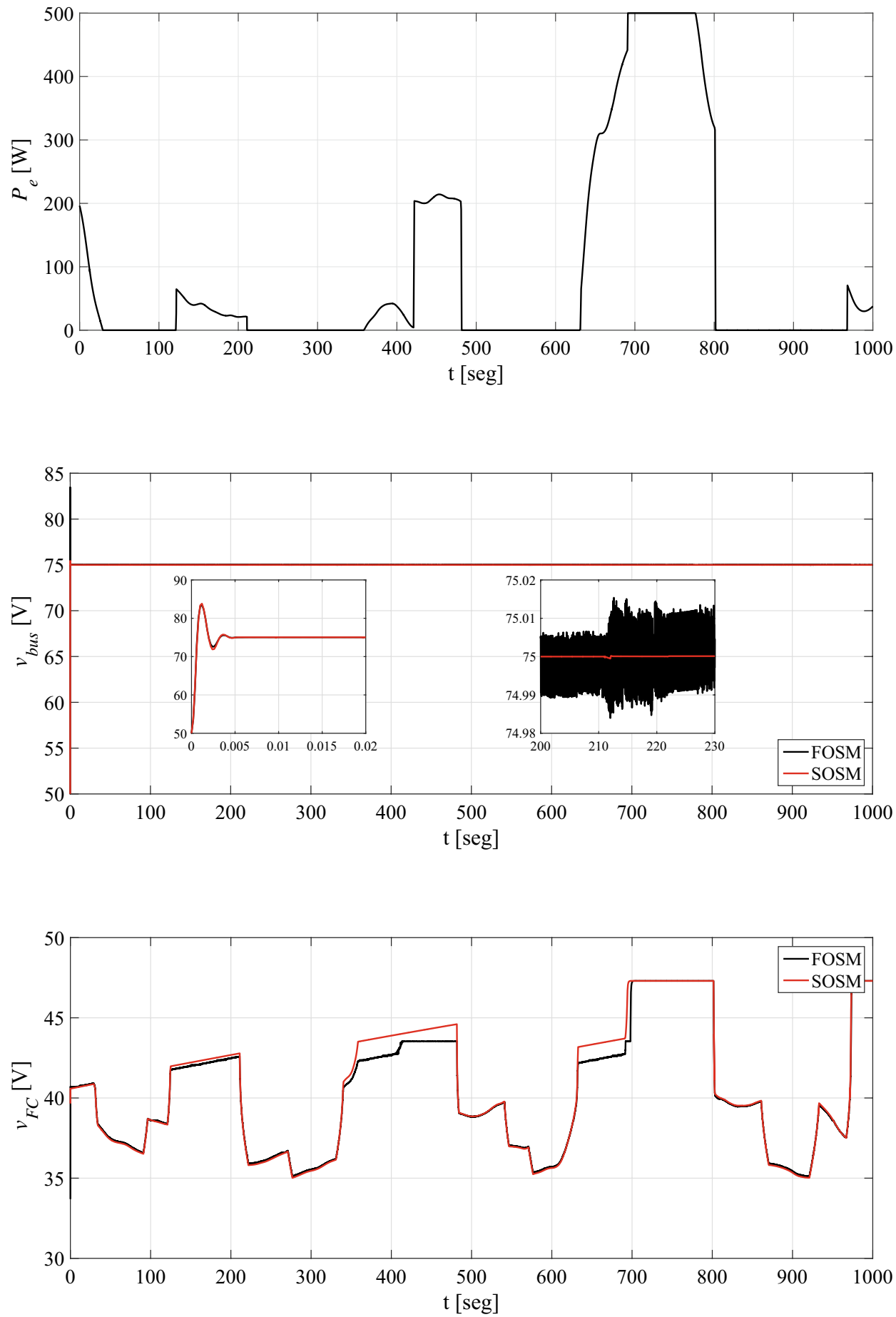
Fig. 11 Fuel cell current $x_{1}=\bar{i}_{\mathrm{FC}}$

Fig. 12 Supercapacitors bank voltage $x_{4}=\bar{v}_{\mathrm{SC}}$

Fig. 13 Supercapacitors bank current $x_{2}=\bar{i}_{\mathrm{SC}}$
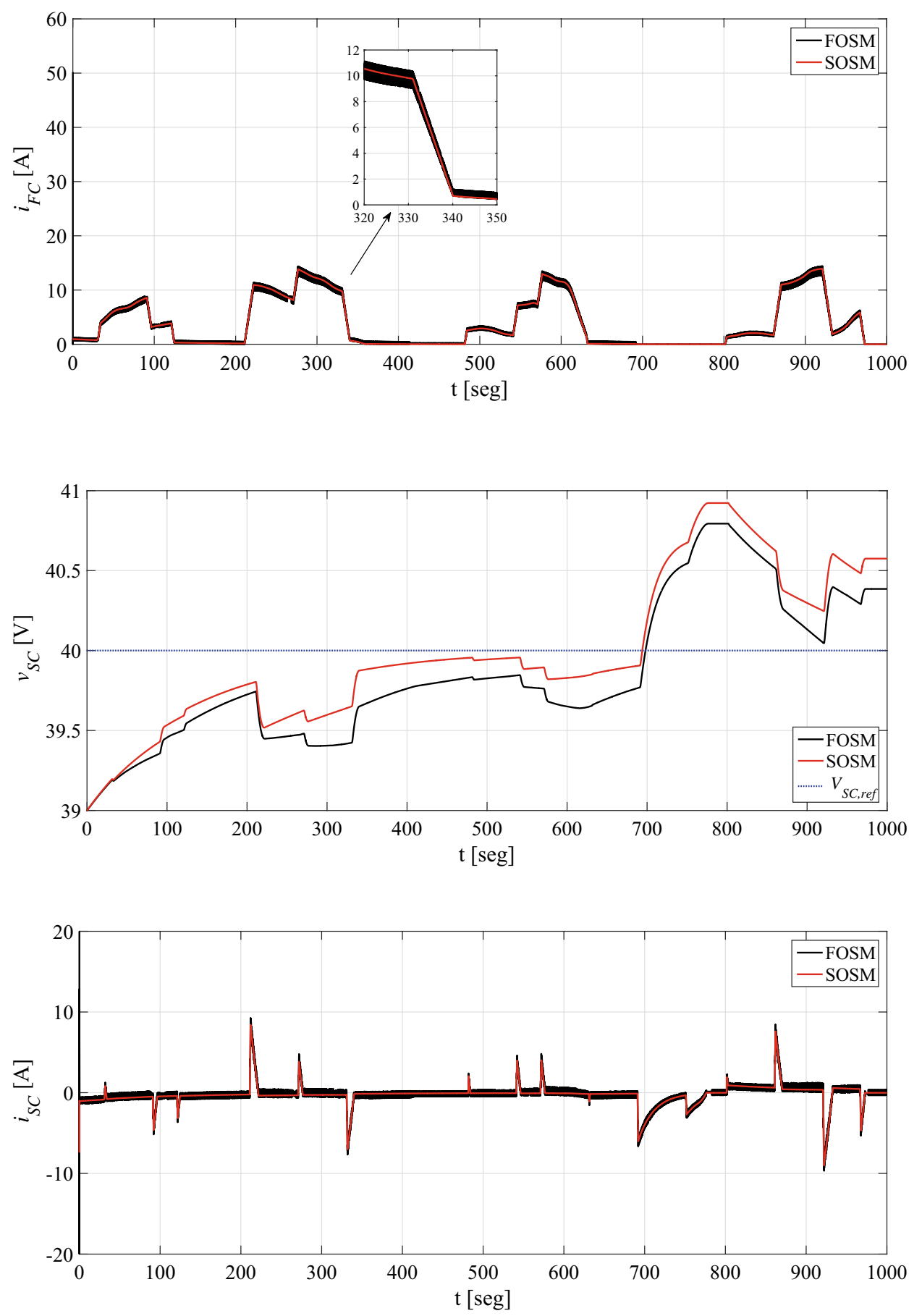

losses in the internal resistances so, as can be seen in Fig. 12, the supercapacitors bank voltage is always lower than in the SOSM case.

Figures 14 and 15 show the switching surfaces for the FOSM and the SOSM controllers. It can be particularly noted the higher chattering of the former at larger load demands (see (20)). Moreover, SOSM presents a neglectable chattering around $s_{i}=0$ (see zoomed area).
Finally, Figs. 16 and 17 display the actual control actions applied to the power converters. It is important to note that these control actions are the ones actually applied to the power converters [i.e., Eq. (9)], computed using Eqs. (14), (15), and (16). As expected, the SOSM controller presents a smooth control action, although the FOSM discontinuous term is considerably reduced using (9), assuring robustness and good perturbation rejection. 
Fig. 14 Switching surface $s_{1}$ (associated with FC converter)
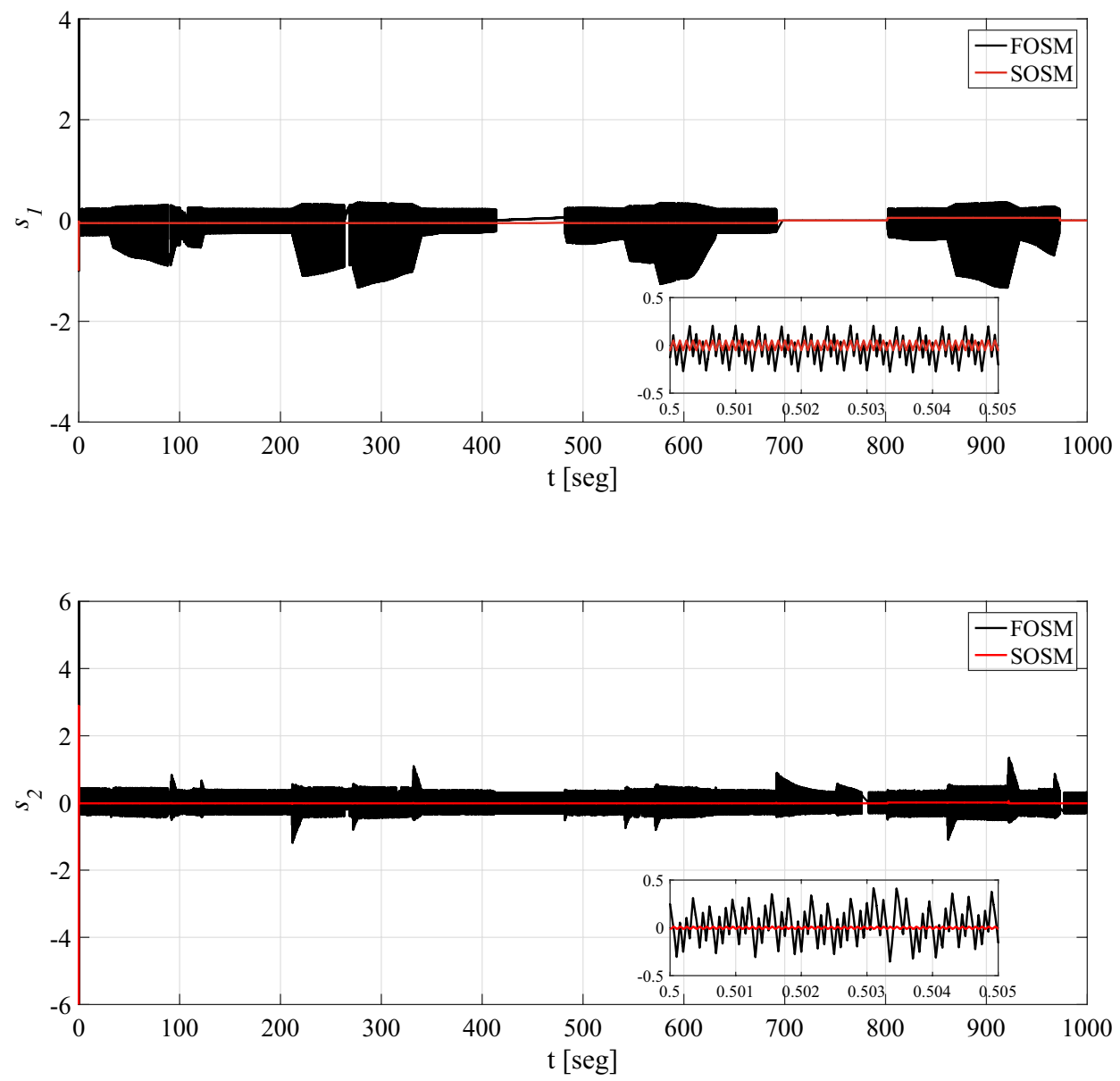

Fig. 15 Switching surface $s_{2}$ (associated with SC converter)

Fig. 16 FC converter actual control action $u_{1}=u_{1 N}+u_{1 \mathrm{MD}}$

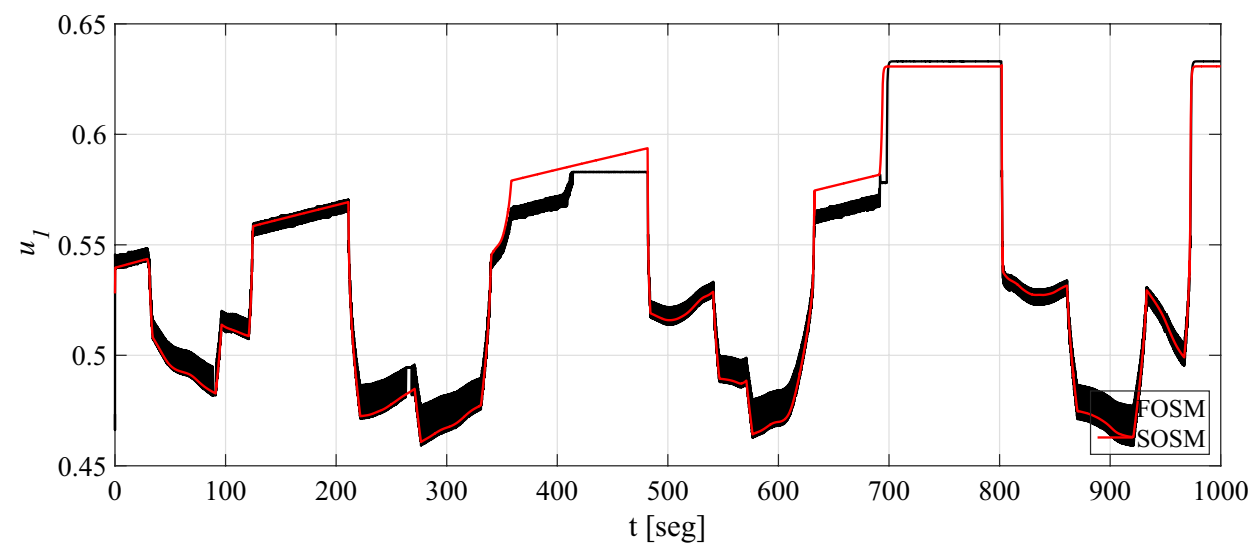

Comparison with classic PID controller

To compare the performance of the designed MIMOsliding mode control setups, simulation results with the most ubiquitous control scheme, namely, Proportional-Integral-Derivative (PID) controller, are presented (most practical feedback loops in industry are PID-type structures). In this case, specifically, one PID controller was designed for the FC power converter to regulate its current. Besides, for the SC boost power converter, two PID controllers were used in nested loops, the inner one to regulate its current and the outer one to regulate the DC bus voltage. The PIDs were, initially, tuned using Ziegler-Nichols methods and, then, were further adjusted through extensive simulation.

The DC bus voltage of the system under abrupt load variations is shown in Fig. 18. Even though the three controllers show a good performance, it should be noted that the PID controllers are slower than both FOSM and SOSM. Particularly, FOSM exhibits the fastest recovery time, but with 
the highest under/overshoot (see left zoomed area) and high chattering. On the other hand, SOSM shows and intermediate recovery time, less under/overshoot and neglectable chattering. It is important to notice also that while the FC current is slowly changing (see right zoomed area), the PID controllers are not able to accurately regulate the DC bus voltage.

Fig. $17 \mathrm{SC}$ converter actual control action $u_{2}=u_{2 N}+u_{2 \mathrm{MD}}$

Fig. 18 DC bus voltage $x_{3}=\bar{v}_{\text {bus }}$ under step-like load power changes

Fig. 19 DC bus voltage $x_{3}=\bar{v}_{\text {bus }}$ under sinusoidal load power changes

\section{power changes}
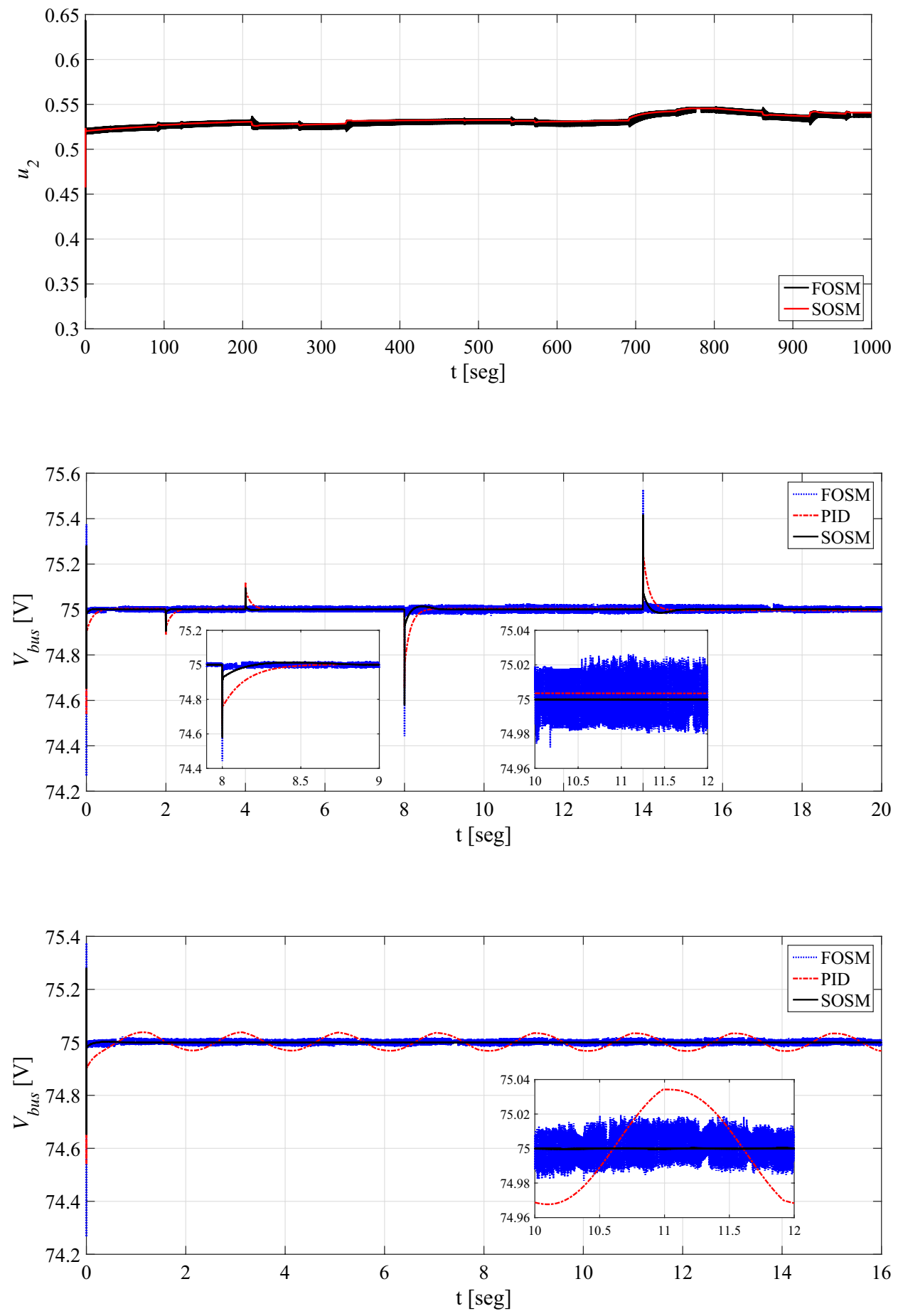

In Fig. 19, a sinusoidal load variation was applied to the system. In this case, both FOSM and SOSM are able to reject this perturbation, while the PID controllers are not able of regulate the DC bus voltage. Although both sliding mode techniques are able to enforce the DC bus voltage, it is important to mention that with FOSM, voltage shows a small modulation around the reference voltage (see zoomed area), while this effect is not present with the SOSM algorithm. por

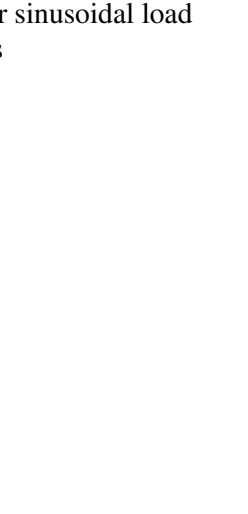


As can be appreciated from these results, the PID controller is unable to accurately reject some perturbations, although its response under step-like load variations may be equivalent to the ones of FOSM and SOSM. On the other hand, both FOSM and SOSM show a considerably better disturbance rejection and with comparable computational cost.

\section{Conclusions}

Two different MIMO sliding mode approaches, namely, FOSM and SOSM, were utilized to tackle the robust control of a fuel cell/sSupercapacitor module intended for a hybrid system. In both cases, the proposed control actions for the $\mathrm{FC}$ and the SC power converters comprised two terms. The first one is a nominal control action, resulting from a simplified equivalent control computation. The second one, in the FOSM case, is a variable-gain SM term, while in the SOSM case is a super-twisting-based control term.

The use of a decoupling diffeomorphism proved to be a convenient method to simplify the sliding mode control design of this FC/SC topology, which is inherently coupled due to the connection of their components to a common DC bus. The diffeomorphism generates a new set of auxiliary control inputs which are decoupled with respect to the sliding variables.

Both proposed MIMO controllers obtained in this way demonstrated to be suitable solutions to robustly fulfil the flow management requirements of the FC/SC module embedded in a HS. Their performances were intensively assessed through realistic in-silico tests, dealing with varying wind and abrupt load demand changes, and extensive model uncertainties.

From comparative analysis, it was established that faster voltage drop recovery time could be achieved with the variable-gains FOSM controller, but with appreciable chattering. On the other hand, the SOSM exhibited slower recovery time, but with neglectable chattering. This can be observed in the DC bus voltage, and the FC and the SC currents, but more importantly, in the switching surfaces and control actions applied to the power converters.

Further comparisons, in this case against classic PID controllers, allowed to conclude that both developed MIMO controllers setups, FOSM and SOSM, displayed better disturbance rejection and performance under abrupt and sinusoidal load variations than the PID structure. Besides, it is worthy to note that the online computational burden of the formers is not exceedingly larger than the one of the PID algorithms.

In addition, it was formally demonstrated in the paper that the FC/SC module, controlled with the proposed MIMO SM setups, is stable. This could be established by studying the zero dynamics, and analysing the equilibrium point and the resultant vector field in the operation range.

Finally, it can be remarked that the MIMO SM controllers setups developed for the FC/SC turns this subsystem into a versatile module, with enough flexibility to be used in different hybrid topologies. For instance, those that incorporates photovoltaic panels, lithium batteries or flow batteries, to name a few.

Acknowledgements This research was supported by the Universidad Nacional de La Plata (UNLP), the CONICET and the ANPCyT, from Argentina. Authors also want to thank the support of the Fuel Cell Group at IRI (CSIC-UPC) and of the institute IOC at the Universitat Politècnica de Catalunya, Barcelona, España.

\section{Compliance with ethical standards}

Conflict of interest On behalf of all authors, the corresponding author states that there is no conflict of interest.

Open Access This article is distributed under the terms of the Creative Commons Attribution 4.0 International License (http://creativeco mmons.org/licenses/by/4.0/), which permits unrestricted use, distribution, and reproduction in any medium, provided you give appropriate credit to the original author(s) and the source, provide a link to the Creative Commons license, and indicate if changes were made.

\section{Appendix}

System parameters nominal values, references, and design gains: $L_{\mathrm{FC}}=35 \mu \mathrm{Hy} ; L_{\mathrm{SC}}=35 \mu \mathrm{Hy} ; C_{\mathrm{bus}}=2720 \mu \mathrm{F}$; $C_{\mathrm{SC}}=165 \mathrm{~F} ; V_{\text {bus }_{\text {ref }}}=75 \mathrm{~V} ; V_{\mathrm{SC}_{\text {ref }}}=40 \mathrm{~V} ; E_{\mathrm{oc}_{\mathrm{L}}}=41,5 \mathrm{~V}$; $R_{\mathrm{FC}_{\mathrm{L}}}=0,3 \Omega ; a_{1} \stackrel{\text { ref }}{=} 1 ; a_{2}=20$.

\section{References}

1. Kunusch, C., Puleston, P.F., Mayosky, M.A.: Sliding Mode Control of PEM Fuel Cells. Springer, Berlin (2012)

2. Saini, P.K., Biswas, A., Bhanja, D.: Performance evaluation and simulation of solar panel. Wind mill, fuel cell hybrid system for small scale energy harvesting. J. Clean Energy Technol. 3(6), 417 (2015)

3. Özgirgin, E., Devrim, Y., Albostan, A.: Modeling and simulation of a hybrid photovoltaic (PV) module-electrolyzer-PEM fuel cell system for micro-cogeneration applications. Int. J. Hydrog. Energy 40(44), 15336 (2015). https://doi.org/10.1016/j. ijhydene.2015.06.122. (The 4th International Conference on Nuclear and Renewable Energy Resources (NURER2014), 26-29 October 2014, Antalya, Turkey)

4. Sedghisigarchi, K., Davari, A., Famouri, P.: Dynamic modeling and control of a fuel cell for electric vehicle applications. In: Vehicle Power and Propulsion Conf. (VPPC), IEEE, pp. 1-5 (2011). https://doi.org/10.1109/VPPC.2011.6043131

5. Torreglosa, J.P., García, P., Fernández, L.M., Jurado, F.: Energy dispatching based on predictive controller of an 
off-grid wind turbine/photovoltaic/hydrogen/battery hybrid system. Renew. Energy 74, 326 (2015). https://doi.org/10.1016/j. renene.2014.08.010

6. Edwards, R.L., Demuren, A.: Regression analysis of PEM fuel cell transient response. Int. J. Energy Environ. Eng. 7(3), 329 (2016). https://doi.org/10.1007/s40095-016-0209-1

7. Cheng, Y.: Assessments of energy capacity and energy losses of supercapacitors in fast charging-discharging cycles. IEEE Trans. Energy Convers. 25(1), 253 (2010). https://doi.org/10.1109/ TEC.2009.2032619

8. Rouholamini, M., Mohammadian, M.: Energy management of a grid-tied residential-scale hybrid renewable generation system incorporating fuel cell and electrolyzer. Energy Build. 102, 406 (2015). https://doi.org/10.1016/j.enbuild.2015.05.046

9. Thomas, J.: Analytical non-linear model predictive control for hybrid systems with discrete inputs only. IET Control Theory Appl. 6(8), 1080 (2012). https://doi.org/10.1049/iet-cta.2010.0675

10. Dey, S., Dash, R., Swain, S.: Fuzzy based optimal load management in standalone hybrid solar PV /wind/fuel cell generation system. In: International Conference on Communication, Control and Intelligent Systems (CCIS), pp. 486-490 (2015)

11. Kraa, O., Saadi, R., Becherif, M., Ayad, M.Y.: Flatness and sliding mode based controller of fuel cell and supercapacitors hybrid source. In: 2015 3rd International Conference on Control, Engineering Information Technology (CEIT), pp. 1-6 (2015). https:// doi.org/10.1109/CEIT.2015.7233183

12. Shtessel, Y., Edwards, C., Fridman, L., Levant, A.: Sliding Mode Control and Observation. Birkhäuser, Boston (2014)

13. Fridman, F.P.Leonid, Barbot, Jean-Pierre: Recent Trends in Sliding Mode Control. IET, London (2016)

14. Li, S., Yu, X., Fridman, L., Man, Z., Wang, X.: Advances in Variable Structure Systems and Sliding Mode Control-Theory and Applications. Springer International Publishing, Berlin (2018)

15. Derbeli, M., Farhat, M., Barambones, O., Sbita, L.: Control of PEM fuel cell power system using sliding mode and super-twisting algorithms. Int J Hydrog Energy 42(13), 8833 (2017). https ://doi.org/10.1016/j.ijhydene.2016.06.103. (Hydrogen Fuel Cell \& Renewable Energy Techniques: The 8th International Conference on Renewable Energy (CIER-2015), 21-23 December 2015, Sousse, Tunisia)

16. Kraa, O., Ghodbane, H., Saadi, R., Ayad, M., Becherif, M., Aboubou, A., Bahri, M.: Energy management of fuel cell/ supercapacitor hybrid source based on linear and sliding mode control. Energy Proc. 74, 1258 (2015). https://doi.org/10.1016/j.egypr o.2015.07.770. (The Int. Conf. on Tech. and Materials for Ren. Energy, Environment and Sustainability (TMREES15))

17. Ashok, R., Shtessel, Y.: Control of fuel cell-based electric power system using adaptive sliding mode control and observation techniques. J. Frankl. Inst. 352(11), 4911 (2015). https://doi. org/10.1016/j.jfranklin.2015.04.010

18. Ashok, R.S., Shtessel, Y.B., Ghanes, M.: Sliding mode control of hydrogen fuel cell and ultracapacitor based electric power system: electric vehicle application. IFAC-Pap. OnLine 50(1), 14794 (2017). https://doi.org/10.1016/j.ifacol.2017.08.2552. 20th IFAC World Congress

19. More, J., Puleston, P., Kunusch, C., Allue, F.: Development and implementation of a supervisor strategy and sliding mode control setup for fuel-cell-based hybrid generation systems. IEEE Trans. Energy Convers. PP(99), 1 (2015). https://doi.org/10.1109/ TEC.2014.2354553

20. Guezennec, Y., Choi, T., Paganelli, G., Rizzoni, G.: Supervisory control of fuel cell vehicles and its link to overall system efficiency and low-level control requirements. In: Proceedings of the American Control Conference. Denver, Colorado (2003)

21. Utkin, V., Gulder, J., Shi, J.: Sliding mode Control in ElectroMechanical Systems. Taylor and Francis, London (1999)

22. Sira-Ramirez, H.: Differential geometric methods in variable structure control. Int. J. Control 48(5), 1359 (1988)

Publisher's Note Springer Nature remains neutral with regard to jurisdictional claims in published maps and institutional affiliations. 